\title{
2,3-Dichloro-5,6-dicyanobenzoquinone (DDQ) mediated oxidation- dehydrogenation of 2-aroyl-3,4-dihydro-2H-benzopyrans : synthesis of 2-aroylbenzopyran-4-ones
}

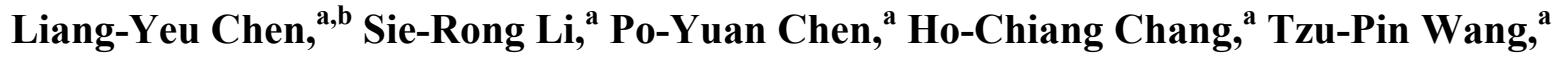 \\ Ian-Lih Tsai, ${ }^{b}$ and Eng-Chi Wang, ${ }^{a, *}$ \\ ${ }^{a}$ Department of Medicinal and Applied Chemistry \\ ${ }^{b}$ Graduate Institute of Pharmaceutical Sciences, Kaohsiung Medical University, Kaohsiung City, \\ 807 Taiwan \\ E-mail: enchwa@kmu.edu.tw
}

\begin{abstract}
DDQ mediated an oxidation-dehydrogenation of 2-aroyl-3,4-dihydro-2H-benzopyran prepared from salicylaldehyde to yield 2-aroylbenzopyran-4-one. Stochiometric amount of DDQ, acetic acid and water in refluxing dioxane are the optimal condition for this oxidation-dehydrogenation reaction. A rationally proposed mechanism for this oxidation-dehydrogenation process is based on the isolation of a reaction intermediate viz., 2-benzoyl-8-methoxy-3,4-dihydrobenzopyran-4one and the identification of the ${ }^{18} \mathrm{O}$-labled carbonyl group of benzopyran-4-one demonstrating incorporation of labeled $\mathrm{H}_{2}{ }^{18} \mathrm{O}$.
\end{abstract}

Keywords: 2-Aroyl-3,4-dihydro-2H-benzopyrans; 2-aroylbenzopyran-4-ones, DDQ, oxidationdehydrogenation

\section{Introduction}

DDQ (2,3-dichloro-5,6-dicyanobenzoquinone) is a chemical reagent widely used in organic synthesis. The major functions of DDQ include the deprotection of thioacetals, acetals and ketals and conversion to aldehydes and ketones, ${ }^{1}$ and the deprotection of benzyl, MPM (4methoxybenzyl) and DMPM (3,4-dimethoxybenzyl) groups into hydroxy groups, ${ }^{2}$ the mediated carbon-carbon bond formation for the synthesis of quinolines from imines and alkynes or alkenes, ${ }^{3}$ the catalyzed benzylic acetoxylation of arylalkanes, ${ }^{4}$ the synthesis of methoxylated $(E)$ arylalkenes and arylalkanones, ${ }^{5}$ the mediation of direct cross-dehydrogenative-coupling (CDC) between ethers and active methylene compounds, ${ }^{6 \mathrm{a}}$ or simple ketones, ${ }^{6 \mathrm{~b}}$ the oxidative cyclization of phenolic Schiff base for the synthesis of 2-arylbenzoxazoles, ${ }^{7}$ the facile synthesis of vicinal diamines via oxidation of the $N$-phenyltetrahydroisoquinolines, ${ }^{8}$ the oxidation of beta-asarone to 
phenylpropanoids, ${ }^{9}$ and the oxidation of arylpropanes to (E)-cinnamaldehydes, ${ }^{10}$ as well as others. ${ }^{11}$ On the other hand, 1-benzopyran-4-one (1-chromen-4-one) is a core structure of isoflavones and flavones which are notable for their biological activities. ${ }^{12}$ However, the synthesis of 2-aroylbenzopyran-4-ones has received little attention. The methods reported include oxidation of benzylchromones with $\mathrm{SeO}_{2},{ }^{13 a}$ and the reaction of chromonecarbonyl chlorides with benzene catalyzed by aluminum chloride to yield the biologically active 2aroylchromen-4-ones. ${ }^{13 \mathrm{~b}, 13 \mathrm{c}}$ The tedious reaction condition, and absence of straightforward protocols are shortcoming of the reported methods. We recently reported on our use of DDQ to oxidize; chromene to chromen-2-one (coumarin), ${ }^{14}$ 4-hydroxychroman to chromen-4-one, ${ }^{15}$ and 5,6-dimethoxy-3-methyl-2-aroylchroman to 5,6-dimethoxy-3-methyl-2-aroylchromen-4-one. ${ }^{16}$ Despite a number of examples that have been reported on the oxidation-dehydrogenation mediation by DDQ, a detailed study on the optimal reaction conditions and mechanism are still outstanding. Herein, we report on an extension of our preliminary results, ${ }^{17}$ on the efficient oxidation of chroman to chromen-4-one by DDQ and provide a rational mechanism deduced by the isolation of a key reaction intermediate as well as the final product which incorporated the ${ }^{18} \mathrm{O}$ when acetic acid and $\mathrm{H}_{2}{ }^{18} \mathrm{O}$ were used in the reaction.

\section{Results and Discussion}

A model reaction was designed for determining the optimal condition for this DDQ oxidation. From careful investigations (Table 1), we found that DDQ (3.5 equv.) in the refluxing dioxane and with $\mathrm{HOAc}-\mathrm{H}_{2} \mathrm{O}$ as an additive (entry 4) are the optimized conditions. Omission of HOAc$\mathrm{H}_{2} \mathrm{O}$ as addictive (entry 3), increases the reaction time and lowers the yield. Using one or two equivalents DDQ (entries 1 and 2) in this oxidation reaction results in no product being observed. This result indicates that the stochiometric amounts of DDQ are necessary in this oxidation reaction. In DMF as solvent and HOAc (few drops) as additive, the reaction takes place but purification is tedious (entry 5). Moreover, with EtOAc as solvent and HOAc as additive, the reaction occurs but a longer reaction time is required (entry 6). This result points to the fact that this type of oxidation requires higher boiling point solvents than that of EtOAc. If only HOAc is used as solvent without $\mathrm{H}_{2} \mathrm{O}$ as an additive, the reaction time is a little longer (entry 7). In the case of using $\mathrm{H}_{2} \mathrm{O}$ as solvent, without HOAc as additive, the yield of product is lower with most of starting material being recovered (entry 8). This result implies that HOAc is a requisite for the enhanced rate of this oxidation. When DMSO was used as solvent under refluxing conditions, the product mixture is difficult to purify resulting in a lower yield (entry 9). Employing the optimized conditions, DDQ (3.5 equv.) in the refluxing dioxane and $\mathrm{HOAc}-\mathrm{H}_{2} \mathrm{O}$ as an additive, a series of 2-aroyl-3,4-dihydro-2H-benzopyrans prepared from salicylaldehyde were oxidized with DDQ to yield the title compounds in moderate to good yield. 
Table 1. The results of the oxidation of $\mathbf{1 b}(2 \mathrm{mmol})$ with DDQ under various conditions in refluxing solvent $(4 \mathrm{~mL})$ to yield $\mathbf{2 b}$



\begin{tabular}{|c|c|c|c|c|c|c|c|c|c|}
\hline Entry & DDQ/Solvent & Additive & $\mathrm{T}^{\mathrm{a}}$ & $\mathrm{Y}^{\mathrm{b}}$ & Entry & DDQ/Solvent & Additive & $\mathrm{T}^{\mathrm{a}}$ & $\mathrm{Y}^{\mathrm{b}}$ \\
\hline 1 & 1 equv/dioxane & - & 12 & $\begin{array}{l}\mathrm{N} \\
\mathrm{D}^{\mathrm{c}}\end{array}$ & 6 & $3.5 \mathrm{equv} / \mathrm{EA}$ & $\mathrm{HOAc}^{\mathrm{b}}$ & 6 & 82 \\
\hline 2 & 2 equv/dioxane & - & 12 & $-d$ & 7 & $\begin{array}{c}3.5 \\
\text { equv/HOAc }\end{array}$ & - & 6 & 81 \\
\hline 3 & $\begin{array}{c}3.5 \\
\text { equv/dioxane }\end{array}$ & - & 12 & 75 & 8 & 3.5 equv $/ \mathrm{H}_{2} \mathrm{O}$ & - & 12 & 32 \\
\hline 4 & $\begin{array}{c}3.5 \\
\text { equv/dioxane }\end{array}$ & $\begin{array}{c}\mathrm{HOAc}^{\mathrm{e}} \\
\mathrm{H}_{2} \mathrm{O}\end{array}$ & 4 & 86 & 9 & $\begin{array}{c}3.5 \\
\text { equv/DMSO }\end{array}$ & $-g$ & 2 & 25 \\
\hline 5 & 3.5 equv/DMF & $\mathrm{HOAc}^{\mathrm{f}}$ & 2 & 80 & & & & & \\
\hline
\end{tabular}

${ }^{\mathrm{a}} \mathrm{T}=$ Reaction time in hours; ${ }^{\mathrm{b}} \mathrm{Y}=\%$ Yield; ${ }^{\mathrm{c}} \mathrm{ND}=$ non-detective; ${ }^{\mathrm{d}}$ incomplete oxidation; ${ }^{\mathrm{e}} \mathrm{HOAC}(0.2 \mathrm{~mL}), \mathrm{H}_{2} \mathrm{O}$ (1-2 drops) in $2 \mathrm{mmol}$ reaction scale, see experimental section; ${ }^{\mathrm{f}}$ Reaction time is shorter but purification is difficulty; ${ }^{\mathrm{g}}$ Product together with the messy by-products were observed.

Structural assignment of 2-benzoyl-8-methoxybenzopyran-4-one $\mathbf{2 b}$ is based on the following spectral data. A three-proton singlet at $\delta 3.98$ in the ${ }^{1} \mathrm{H}-\mathrm{NMR}$ spectrum is assigned to the 8-methoxy group while the NOESY spectrum illustrates the following; a one proton double doublet signal at $\delta 7.25(\mathrm{dd}, J=8.0,1.2 \mathrm{~Hz})$ cross linked to the methoxy group can be assigned to H-7; cross linking between H-6 and H-7 and H-5 with H-6 allows the signals for H-6 at $\delta 7.39$ (t, $J=8.4 \mathrm{HZ}$ ) and $\mathrm{H}-5$ at $\delta 7.78(\mathrm{dd}, J=8.2,1.2 \mathrm{~Hz})$ to be assigned. Based on the HSQC technique, C-3 at $\delta 114.6$ is shown linked to $\mathrm{H}-3$ at $\delta 6.92$; $\mathrm{C}-7$ at $\delta 115.1$ is shown linked to $\mathrm{H}-7$ at $\delta$ 7.25; C-5 at $\delta 116.4$ is shown linked with $\mathrm{H}-5$ at $\delta 7.78$ and $\mathrm{C}-6$ at $\delta 125.7$ is shown linked to $\mathrm{H}-6$ at $\delta$ 7.37. Based on the HMBC technique, the (C-4) carbonyl carbon at $\delta 146.3$ shows cross links with H-3 and H-5 while the benzoyl carbon at $\delta 149.3$ shows cross links with $\mathrm{H}-3, \mathrm{H}-$ 2', and H-6'. The DEPT ${ }^{13} \mathrm{C}-\mathrm{NMR}$ spectra for $\mathbf{2 b}$ illustrates the following; in a broad banddecouple spectrum, which shows signals for 15 carbons; in DEPT-90 spectrum, which shows signals for seven $\mathrm{CH}$ carbons and one $\mathrm{CH}_{3}$ carbon; and in DEPT-135 spectrum, which shows seven $\mathrm{CH}$ positive signals and shows no negative signals of $\mathrm{CH}_{2}$. All DEPT ${ }^{13} \mathrm{C}$-NMR spectra 
which are obtained further support the correctness of structure 2b. Additionally EI-MS and EA data are consistent with the structure.

In order to clarify and establish the detailed reaction mechanism, an optimized reaction was quenched after $3 \mathrm{~h}$. The resultant intermediate which was analyzed by spectral analysis ${ }^{1} \mathrm{H}-\mathrm{NMR}$, ${ }^{13} \mathrm{C}-\mathrm{NMR}$, and EI-MS, proved to be 2-benzoyl-8-methoxychroman-4-one $\mathbf{2 b - 1}$ and was shown to be further oxidized to yield $\mathbf{2 b}$ under the same reaction conditions. This result implies that the chroman ring is firstly oxidized to chroman-4-one and then followed by dehydrogenation to yield chromen-4-one. Moreover, in order to clarify the origin of the oxygen of chroman-4-one as well as the involvement of $\mathrm{H}_{2} \mathrm{O}$ in the oxidation reaction, $\mathrm{H}_{2} \mathrm{O}^{18}$ was used at the beginning of reaction using the optimized condition to determine if it was incorporated. The products we isolated proved to be a mixture of the labeled 2-benzoyl-8-methoxybenzopyran-4-one $\left({ }^{18} \mathrm{O}\right)\left(\mathbf{2} \mathbf{b}-{ }^{18} \mathrm{O}\right)$ and the unlabeled one $\mathbf{2 b}$. The labeled benzopyran-4-one $\left({ }^{18} \mathrm{O}\right)$ can be easily verified and distinguished by comparing the MS spectra with the unlabeled one. Chart 1 illustrates the EI-MS spectrum of unlabeled benzopyran-4-one 2b (HRMS, calcd for $\mathrm{C}_{17} \mathrm{H}_{12} \mathrm{O}_{4}, 280.0736$. Found: 280.0738), and Chart 2 illustrates labeled (HRMS, calcd for $\mathrm{C}_{17} \mathrm{H}_{12} \mathrm{O}_{3}{ }^{18} \mathrm{O}, 282.0778$. Found: 282.0776). The inference of this is that the oxygen atom at $\mathrm{C} 4$ of chroman-4-one may arise from either water or acetate. Water may be present in undried solvent or from environmental moisture. In a previous study of Harvey, R. G. et al., they proposed that benzylic oxidation by DDQ occurred through the addition of an acetate anion to the benzylic cation to form the acetic ester, which was subsequently oxidized to a carbonyl intermediate, ${ }^{18}$ but failed to provide a detailed reaction mechanism.

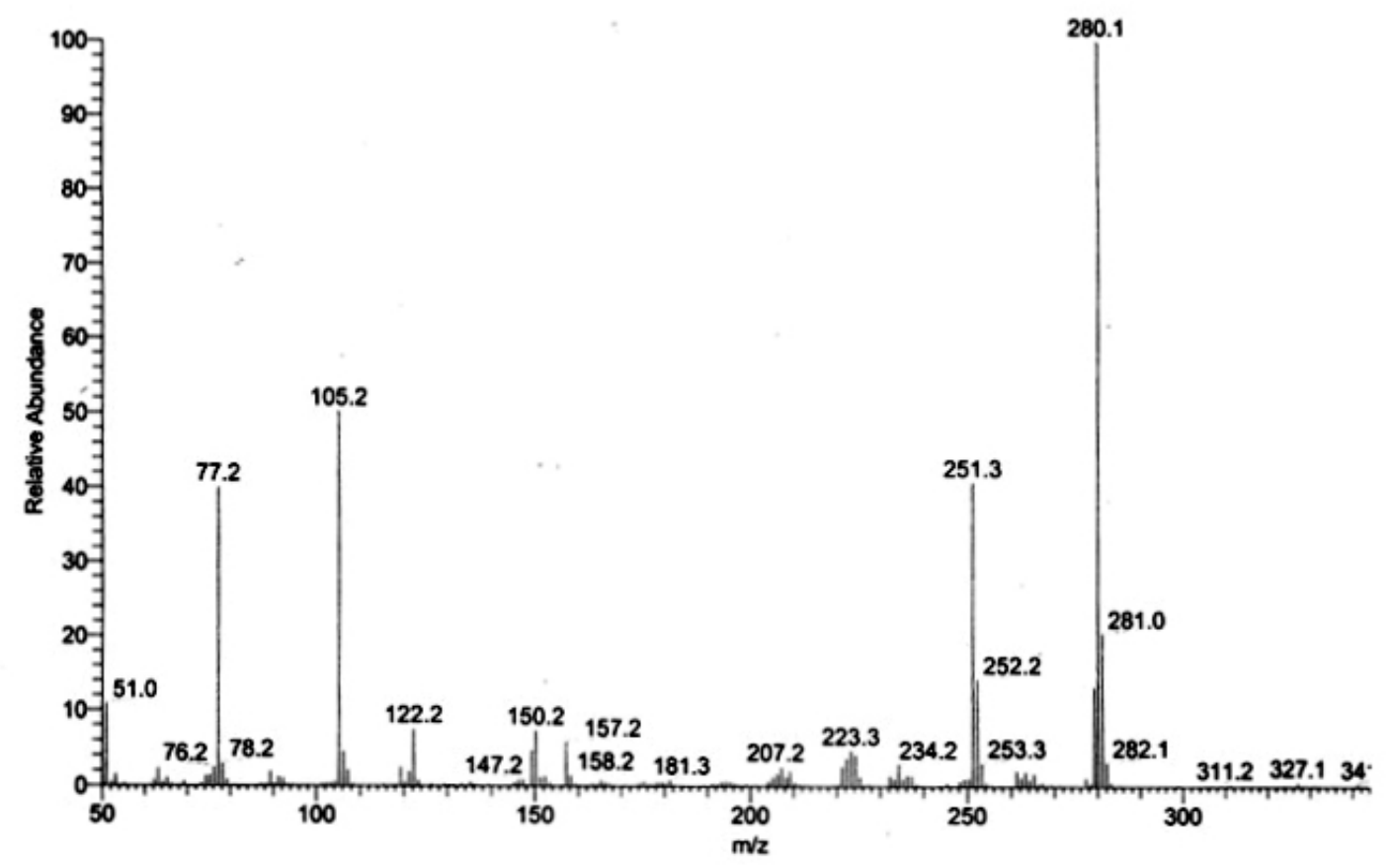

Chart 1. The mass spectrum of pure $\mathbf{2 b}$. 


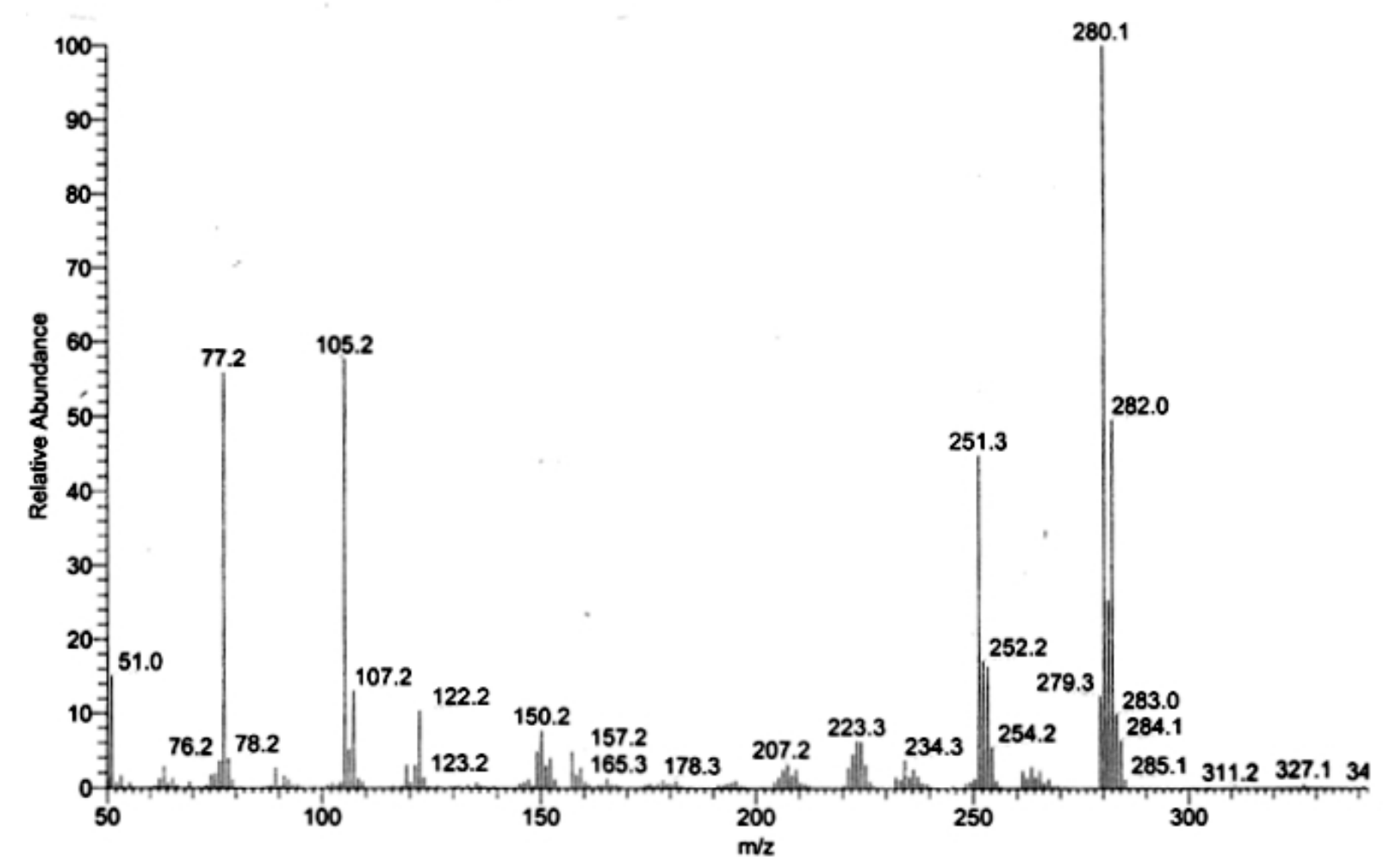

Chart 2. The mass spectrum of the mixture of $\mathbf{2 b}$ and $\mathbf{2 b}-{ }^{18} \mathrm{O}$.

Based on the evidences at hand, we wish to propose a reaction mechanism for this oxidationdehydrogenation process. Using $\mathbf{1 b}$ as an example and depicted as follows (Scheme 1). Initially, one of the benzylic hydrogen of $\mathbf{1 b}$ was abstracted by DDQH ${ }^{+}$which was generated by DDQ and HOAc, to form the benzylic cation $\mathbf{I}$. $\mathrm{DDQH}^{+}$having a positive charge acts as a stronger electrophile than that of neutral DDQ, and thus enhances the deprotonation process of chroman at $\mathrm{C}-4$ position. Following on from this addition of an acetate anion (from acetic acid) or $\mathrm{H}_{2} \mathrm{O}$ (from additive / surrounding / solvent) II-1 and II-2 could be generated, respectively. In this step, II-1 has a higher priority than II-2 since the nucleophilicity of acetate $>\mathrm{H}_{2} \mathrm{O}$. Consequently, II-1 could be transferred into III by hydrolysis with $\mathrm{H}_{2} \mathrm{O}$ and II-2 could be converted to III by deprotonation by DDQ. Reaction between DDQH ${ }^{+}$and III would then form 2-aroyl-3,4-dihydrobenzopyran-4-one VI as an intermediate by going through transiention states IV and V. Subsequently tautomerism of VI to the enolic form VII and attack by DDQH generated between DDQ and $\mathrm{H}_{3} \mathrm{O}^{+}$would yield VIII. After a deprotonation-protonation process, intermediate IX is attacked by water to eliminate a neutral DDQ-2H and finally yield 2-aroylbenzopyran-4-one $\mathbf{2 b}$. In the overall reaction, three equivalents of DDQ are consumed with three equivalent of DDQ-2H being generated. 

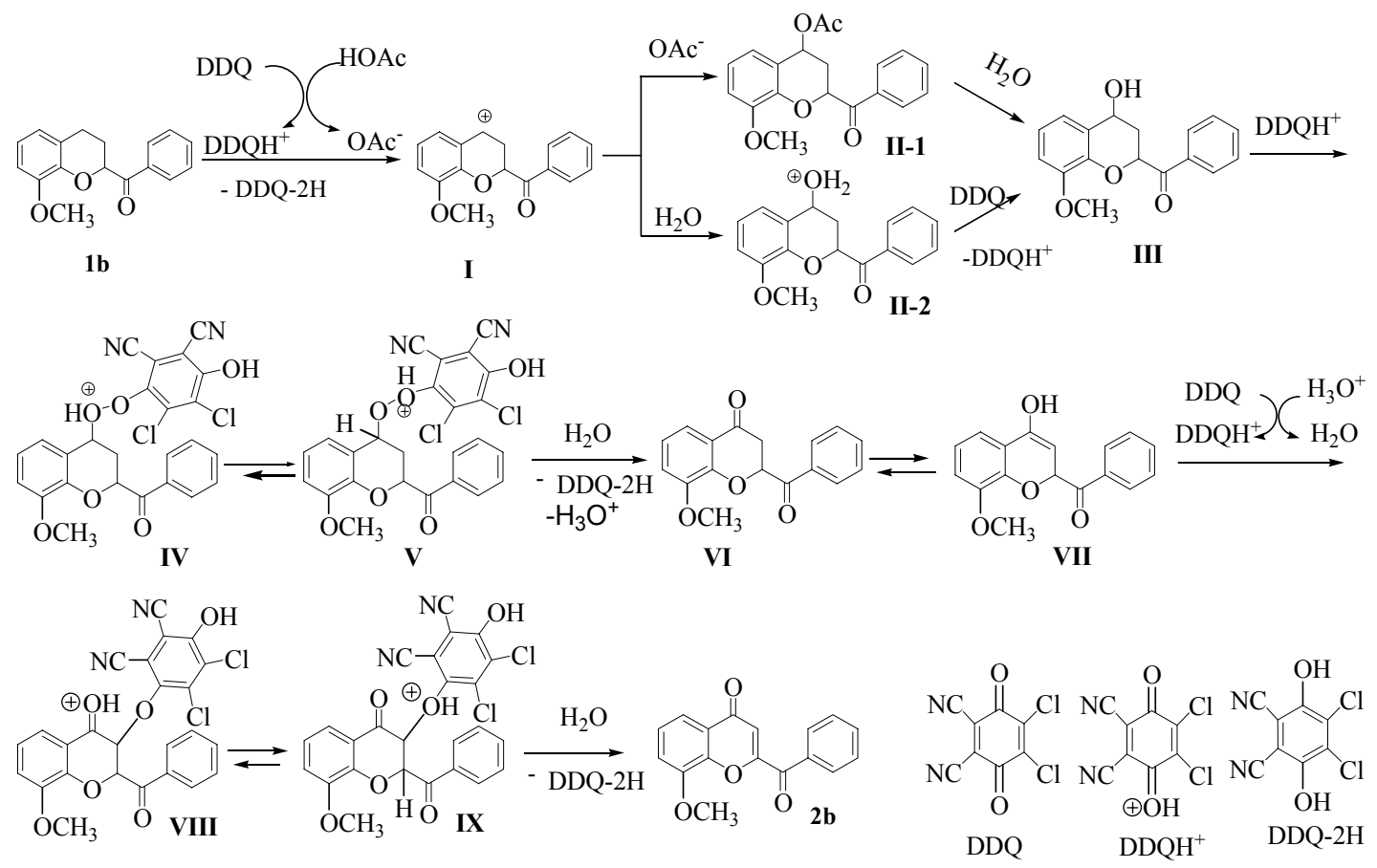

Scheme 1. The proposed mechanism of oxidation-dehydrogenation of chroman to chromen-4one by DDQ.

The proposed mechanism strongly supports the finding that stochiometric amounts of DDQ are required in this oxidation-dehydrogenation reaction. Using the optimal conditions found in this study, 2-aroyl-3,4-dihydro- $2 H$-benzopyrans 1a-j were oxidized to the corresponding 2aroylchromen-4-ones 2a-j using DDQ (3.5 equiv) as oxidant, and $\mathrm{HOAc}-\mathrm{H}_{2} \mathrm{O}$ as additives in refluxing dioxane (Scheme 2). The physical data and selected spectra data of 2a-j are summarized in Table 2.



$$
\begin{aligned}
& \text { a. } R_{1}=R_{2}=R_{3}=R_{4}=H ; \text { b. } R_{1}=R_{2}=R_{4}=H, R_{3}=O M e ; \\
& \text { c. } R_{1}=B r, R_{2}=R_{3}=R_{4}=H ; \text { d. } R_{1}=C l, R_{2}=R_{3}=R_{4}=H ; \\
& \text { e. } R_{1}=R_{2}=H, R_{3}=R_{4}=O M e ; \text { f. } R_{1}=R_{3}=H, R_{2}=R_{4}=O M e ; \\
& \text { g. } R_{1}=R_{3}=C l, R_{2}=R_{4}=H ; \text { h. } R_{1}=R_{4}=C l, R_{2}=R_{3}=H ; \\
& \text { i. } R_{1}=C l, R_{2}=R_{3}=H, R_{4}=O M e ; j . R_{1}=R_{2}=R_{3}=H, R_{4}=O M e
\end{aligned}
$$

Reagents and Conditions: i. DDQ (3.5 equiv), dioxane, $\mathrm{HOAc}-\mathrm{H}_{2} \mathrm{O}$ reflux; $34-86 \%$

Scheme 2. The oxidation of 2-aroyl-3,4-dihydro-2H-benzopyrans 1a-j with DDQ to yield 2aroylbenzopyran-4-ones $\mathbf{2} \mathbf{a}-\mathbf{j}$. 
Table 2. The physical and selected spectral data of $\mathbf{2 a - j}$

\begin{tabular}{|c|c|c|c|c|c|c|c|c|c|}
\hline Compd & MP $\left({ }^{\circ} \mathrm{C}\right)$ & $\begin{array}{c}\% \\
\text { Yield }\end{array}$ & $\begin{array}{c}\text { Reaction } \\
\text { time (hr) }\end{array}$ & IR $\left(\mathrm{cm}^{-1}\right)$ & $\mathrm{H}-3(\delta)$ & $\mathrm{C}-2^{\mathrm{b}}$ & $\mathrm{C}-3^{\mathrm{b}}$ & $\begin{array}{c}\mathrm{C}=\mathrm{O} \\
(\mathrm{C}-4)^{\mathrm{b}}\end{array}$ & $\mathrm{ArC}^{\mathrm{n}}=\mathrm{O}^{\mathrm{b}}$ \\
\hline $\mathbf{2 a}$ & $87-88$ & 76 & 4 & 1655,1615 & 6.85 & 157.8 & 114.8 & 178.1 & 187.8 \\
\hline $\mathbf{2 b}$ & $182-183$ & 86 & 4 & 1677,1647 & 6.92 & 157.8 & 114.5 & 178.2 & 187.2 \\
\hline $\mathbf{2 c}$ & $159-160$ & 68 & 8 & 1654,1597 & 6.89 & 158.0 & 115.0 & 176.9 & 187.6 \\
\hline $\mathbf{2 d}$ & $150-152$ & 70 & 8 & 1657,1598 & 6.89 & 157.9 & 114.8 & 177.0 & 187.5 \\
\hline $\mathbf{2 e}$ & $166-167$ & 82 & 4 & 1646,1599 & 6.92 & 158.6 & 115.0 & 178.3 & 185.3 \\
\hline $\mathbf{2 f}$ & $181-182$ & 85 & 4 & 1648,1600 & 6.78 & 158.1 & 114.8 & 177.4 & 186.2 \\
\hline $\mathbf{2 g}$ & $132-134$ & $34^{\mathrm{a}}$ & 12 & 1661,1595 & 7.02 & 158.3 & 114.4 & 176.4 & 186.5 \\
\hline $\mathbf{2 h}$ & $168-170$ & $48^{\mathrm{a}}$ & 8 & 1651,1607 & 6.89 & 157.7 & 114.7 & 176.9 & 186.3 \\
\hline $\mathbf{2 i}$ & $151-152$ & 65 & 8 & 1655,1598 & 6.84 & 158.8 & 114.2 & 177.0 & 185.7 \\
\hline $\mathbf{2 j}$ & $82-83$ & 73 & 4 & 652,1597 & 6.85 & 158.7 & 114.3 & 178.2 & 186.0 \\
\hline
\end{tabular}

${ }^{a}$ Even prolong reaction time, the \%yield cannot be improved, but recovery starting material. ${ }^{\mathrm{b}}$ The chemical shift of $\mathrm{C}-2, \mathrm{C}-3$, and two $\mathrm{C}=\mathrm{O}$ group are determined by HSQC or HMBC technique.

Considering Table 2 and based simply on the \% yields, the effect of substituents on the reaction outcome demonstrate the following trend, $\mathrm{OCH}_{3}>\mathrm{H}>\mathrm{Br}>\mathrm{Cl}$. For example, the \% yields of $\mathbf{2 b}, \mathbf{2 e}$, and $\mathbf{2} \mathbf{f}$ which have a methoxy group on the benzene and chroman ring are 86, 82 , and $85 \%$ yield, respectively. The \% yield of $2 \mathrm{a}$ which has no substituents on either the benzene or chroman ring is $76 \%$ while $2 \mathbf{d}$ and $2 \mathbf{i}$ which both have a $\mathrm{Cl}$ at $\mathrm{R}_{1}$ are formed in 70 and $65 \%$ yield respectfully. However $2 \mathrm{~g}\left(\mathrm{R}_{1}=\mathrm{R}_{3}=\mathrm{Cl}\right)$ and $2 \mathrm{~h}\left(\mathrm{R}_{1}=\mathrm{R}_{4}=\mathrm{Cl}\right)$, both having two $\mathrm{Cl}$ atoms were only formed in 35 and $49 \%$ yield respectively. These findings support our proposed reaction mechanism since the formation of a benzilic cation in the first step is stabilized by electron-donating groups in the aromatic ring although not directly conjugated except for $\mathbf{2 f}$. 


\section{Conclusions}

We have, by isolating the intermediate 2-aroylchroman-4-one during the DDQ mediated oxidation process, verified that the C-4 of the chroman ring of 2-aroyl-3,4-dihydro- $2 \mathrm{H}$ benzopyrans is initially oxidized to the carbonyl group followed by dehydrogenation to form chromen-4-one. By identifying labeled 2-aroylchromen-4-one $\left({ }^{18} \mathrm{O}\right)$ by EI-MS, the possible role $\mathrm{H}_{2} \mathrm{O}$ plays in this DDQ oxidation is also evident. Furthermore, HOAc as an additive facilitated the DDQ oxidation. Thus, we have optimized conditions for conversion of 2-aroylchromans to 2aroylchromen-4-ones by DDQ in refluxing dioxane with $\mathrm{HOAc}-\mathrm{H}_{2} \mathrm{O}$ as additives.

\section{Experimental Section}

General. All melting points were determined on a Yanaco micro melting-point apparatus. ${ }^{1} \mathrm{H}$ NMR and ${ }^{13} \mathrm{C}$ NMR spectra were obtained on a Varian Unity plus 400 Spectrometer. Chemical shifts are indicated in parts per million with respect to TMS. IR spectra were measured on a Perkin Elmer system 2000 FT-IR spectrometer. Elemental analyses were recorded on a Heraeus CHN-O Rapid analyzer. Mass spectra were recorded on a Chem/hp/middle spectrometer connected to a Hewlett Packard series II model gas- liquid chromatography. HRMS spectra were performed on a JEOL JMS SX/SX 102A instrument. Silica gel (230-400 mesh) for column chromatography and precoated silica gel plates (60 F-254) for TLC was purchased from E. Merck Co. UV light (254 nm) was used to detect spots on TLC plates after development.

\section{Compounds (1a-j) were prepared according to the procedure of our previous report. ${ }^{17}$ General procedure for the preparation of 2-Aroyl-1-benzopyran-4-one (2a-j)}

Compound 1a-j (2 mmol) dissolved in 1,4-dioxane (4 mL) was stirred and added DDQ (1.60 g, 7 mmol), HOAc $(0.2 \mathrm{~mL})$ and $\mathrm{H}_{2} \mathrm{O}$ (1-2 drops). The reaction mixture was heated to the reflux for 4 $-8 \mathrm{~h}$. The resultant mixture was quenched with saturated brine $(20 \mathrm{~mL})$ and was extracted with $\mathrm{CH}_{2} \mathrm{Cl}_{2}(10 \mathrm{~mL} \times 3)$. The organic layers were combined, washed with brine, and dried with anhydrous $\mathrm{MgSO}_{4}$, in sequence. After filtration, the filtrate was concentrated in vacuo. The resultant residue was purified by silica gel column chromatography (ethyl acetate $/ n$-hexane $=1$ : 10) to give pure $\mathbf{2} \mathbf{a}-\mathbf{j}$.

2-Benzoyl-1-benzopyran-4-one (2a). It ( $0.38 \mathrm{~g}, 76 \%)$ was obtained as colorless crystals, mp 91 ${ }^{\circ} \mathrm{C}$ (lit. ${ }^{13 \mathrm{c}} 94{ }^{\circ} \mathrm{C}$ ); $\mathrm{R}_{\mathrm{f}} 0.31$ (ethyl acetate/ $n$-hexane=1:4); IR (neat) $v_{\max } 3066$ (w), 1655 (vs), 1615 (m), 1464 (s), 1379 (s), 1302 (m), 1263 (s), 1240 (s), 1104 (m), 983 (s), 869 (s) cm ${ }^{-1} ;{ }^{1} \mathrm{H}$ NMR $\left(\mathrm{CDCl}_{3}, 400 \mathrm{MHz}\right) \delta 6.85(\mathrm{~s}, 1 \mathrm{H}, \mathrm{H}-3), 7.44(\mathrm{td}, J=8.0,0.8 \mathrm{~Hz}, 1 \mathrm{H}, \mathrm{ArH}), 7.50-7.56(\mathrm{~m}, 3 \mathrm{H}$, ArH), 7.66, (tt, $J=7.6,1.2 \mathrm{~Hz}, 1 \mathrm{H}, \mathrm{ArH}), 7.73$ (ddd, $J=8.4,7.2,1.6 \mathrm{~Hz}, 1 \mathrm{H}, \mathrm{ArH}), 7.93-7.96(\mathrm{~m}$, $2 \mathrm{H}, \mathrm{ArH}), 8.20(\mathrm{dd}, J=8.0,2.0 \mathrm{~Hz}, 1 \mathrm{H}, \mathrm{ArH}) ;{ }^{13} \mathrm{C} \mathrm{NMR}\left(\mathrm{CDCl}_{3}, 100 \mathrm{MHz}\right) \delta{ }^{13} \mathrm{C} \mathrm{NMR}\left(\mathrm{CDCl}_{3}\right.$, $100 \mathrm{MHz}) \delta 114.8$ (d), 118.6 (d), 124.4 (s), 125.7 (d), 125.9 (d), 128.7 (d), 130.0 (d), 134.1 (d), 134.6 (d), 134.7 (s), 155.7 (s), 157.8 (s), 178.1 (s), 187.8 (s); EI-MS (70 eV) m/z (rel. intensity, 
\%) $250\left(\mathrm{M}^{+}, 11\right), 249$ (14), 223 (16), 222 (100), 221 (17), 105 (68), 77 (42); Anal. calcd for $\mathrm{C}_{16} \mathrm{H}_{10} \mathrm{O}_{3}$ : C, 76.79; H, 4.03. Found: C, 76.77; H, 4.06.

2-Benzoyl-8-methoxy-1-benzopyran-4-one (2b). It ( $0.48 \mathrm{~g}$, 86\%) was obtained as a colorless crystals, mp 183-184 ${ }^{\circ} \mathrm{C} ; \mathrm{R}_{\mathrm{f}} 0.18$ (ethyl acetate $/ n$-hexane $=1: 4$ ); IR (neat) $v_{\max } 1677$ (s), 1647 (vs), 1577 (s), 1488 (s), 1451 (s), 1381 (s), 1277 (vs), 1180 (s), 1054 (s), 735 (s) cm ${ }^{-1}$; ${ }^{1} \mathrm{H}$ NMR $\left(\mathrm{CDCl}_{3}, 400 \mathrm{MHz}\right) \delta 3.99\left(\mathrm{~s}, 3 \mathrm{H}, \mathrm{OCH}_{3}\right), 6.94(\mathrm{~s}, 1 \mathrm{H}, \mathrm{H}-3), 7.25$ (dd, $\left.J=8.0,1.2 \mathrm{~Hz}, 1 \mathrm{H}, \mathrm{H}-7\right)$, 7.39 (t, $J=8.0 \mathrm{~Hz}, 1 \mathrm{H}, \mathrm{H}-6$ ), 7.55 (t, $J=8.0,2 \mathrm{H}, \mathrm{H}-5^{\prime}$ 'and H-3'), 7.69 (t, $J=8.0 \mathrm{~Hz}, 1 \mathrm{H}, \mathrm{H}-4$ ') 7.78 (dd, $J=8.0,1.4 \mathrm{~Hz}, 1 \mathrm{H}, \mathrm{H}-5), 8.09$ (dd, $J=8.0,1.2 \mathrm{~Hz}, 2 \mathrm{H}, \mathrm{H}-2$ ' and $\left.\mathrm{H}-6{ }^{\prime}\right) ;{ }^{13} \mathrm{C}^{\mathrm{NMR}}\left(\mathrm{CDCl}_{3}, 100\right.$ $\mathrm{MHz}) \delta 56.4$ (q) $\left(\mathrm{OCH}_{3}\right), 114.6$ (d) (C-3), 115.1 (d) (C-7), 116.4 (d) (C-5), 125.5 (s), 125.7 (d) (C-6), 128.7 (d) (C-3', C-5), 130.3 (d) (C-2', 5'), 134.2 (d) (C-4'), 134.7 (s), 146.3 (s), 149.3 (s), 157.8 (s) (C-2), 178.2 (s) (C-4), 187.2 (s) (Ar'C=O); EI-MS (70 eV) m/z (rel intensity, \%) 280 $\left(\mathrm{M}^{+}, 82\right), 279$ (10), 252 (12), 251 (37), 150 (11), 122 (12), 105 (100), 77 (52); Anal. calcd for $\mathrm{C}_{17} \mathrm{H}_{12} \mathrm{O}_{4}: \mathrm{C}, 72.85 ; \mathrm{H}, 4.32$. Found: $\mathrm{C}, 72.88 ; \mathrm{H}, 4.37$.

At the same reaction condition for preparing $\mathbf{2 b}$ but it was quenched after the reflux for $3 \mathrm{~h}$. Worked up and purified by silica gel column chromatography (ethyl acetate $/ n$-hexane $=1: 10$ ) to give 2-benzoyl-8-methoxychroman-4-one 2b-1. It (0.34 g, 61\%) was obtained as colorless crystals, mp 121-122 ${ }^{\circ} \mathrm{C}, \mathrm{R}_{\mathrm{f}} 0.41$ (EA/n-hexane = 1:3), IR (KBr) cm ${ }^{-1}: 1693$ (vs), 1583 (m), 1488 (s), $1297(\mathrm{~m}), 1264$ (s), 1211 (m), $1182(\mathrm{~m}) ;{ }^{1} \mathrm{H}-\mathrm{NMR}\left(\mathrm{CDCl}_{3}, 400 \mathrm{MHz}\right) \delta 3.08$ (dd, $J=17.0,4.4$ $\mathrm{Hz}, 1 \mathrm{H}, \mathrm{H}-3 \mathrm{a}$ ), 3.18 (dd, $J=17.0,9.2 \mathrm{~Hz}, 1 \mathrm{H}, \mathrm{H}-3 \mathrm{~b}$ ), 3.84 (s, 3H, $\mathrm{OCH}_{3}$ ), 5.89 (dd, $J=9.2 \mathrm{~Hz}, 4.4$ $\mathrm{Hz}, 1 \mathrm{H}, \mathrm{H}-2), 6.98$ (t, $J=8.0 \mathrm{~Hz}, 1 \mathrm{H}), 7.06$ (dd, $J=8.0 \mathrm{~Hz}, 1.6 \mathrm{~Hz}, 1 \mathrm{H}), 7.48-7.52$ (m, 3H, ArH), 7.62 (tt, $J=7.6 \mathrm{~Hz}, 1.2 \mathrm{~Hz}, 1 \mathrm{H}, \mathrm{ArH}), 8.07$ (dd, $J=8.4 \mathrm{~Hz}, 1.2 \mathrm{~Hz}, 1 \mathrm{H}),{ }^{13} \mathrm{C}-\mathrm{NMR}\left(\mathrm{CDCl}_{3}, 100\right.$ MHz) $\delta 39.1$ (t), 56.3 (q), 78.5 (d), 117.5 (d), 118.0 (d), 121.6 (d), 121.8 (s), 128.7 (d), 129.3 (d), 133.9 (s), 134.0 (d), 148.9 (s), 150.2 (s), 190.2 (s), 194.1 (s); EIMS (70 eV) $\mathrm{m} / z$ (rel. intensity, \%) 282 ([M] $\left.]^{+}, 75\right), 177$ (69), 162 (29), 161 (85), 160 (85), 149 (17), 121 (30), 105 (100), 77 (61), 51(15); Anal. calcd for $\mathrm{C}_{17} \mathrm{H}_{14} \mathrm{O}_{4}$ : C, 72.33; H, 5.00. Found: C, 72.31; H, 4.98.

2-Benzoyl-6-bromo-1-benzopyran-4-one (2c). It $(0.45 \mathrm{~g}, 69 \%)$ was obtained as colorless crystals, mp $161-162{ }^{\circ} \mathrm{C}\left(\right.$ lit. ${ }^{13 \mathrm{c}} 161{ }^{\circ} \mathrm{C}$ ); $\mathrm{R}_{\mathrm{f}} 0.46$ (ethyl acetate $/ n$-hexane $=1: 4$ ); IR (neat) $v_{\max }$ 1654 (vs), 1597 (m), 1462 (s), 1433 (s), 1358 (m), 1271 (s), 1236 (m), 984 (m), 726 (m) cm ${ }^{-1} ;{ }^{1} \mathrm{H}$ NMR $\left(\mathrm{CDCl}_{3}, 400 \mathrm{MHz}\right) \delta 6.89$ (s, 1H, H-3), 7.49 (d, $\left.J=8.8 \mathrm{~Hz}, 1 \mathrm{H}, \mathrm{H}-8\right), 7.54-7.58$ (m, 2H, H3' and H-5'), 7.71 (tt, $J=7.6,1.2 \mathrm{~Hz}, 1 \mathrm{H}, \mathrm{H}-4^{\prime}$ ), 7.84 (dd, $J=8.8,2.4 \mathrm{~Hz}, 1 \mathrm{H}, \mathrm{H}-7$ ), 7.94-7.97 (m, $2 \mathrm{H}, \mathrm{H}-2^{\prime}$ and $\mathrm{H}-6$ '), $8.36(\mathrm{~d}, J=2.4 \mathrm{~Hz}, 1 \mathrm{H}, \mathrm{H}-5) ;{ }^{13} \mathrm{C} \mathrm{NMR}\left(\mathrm{CDCl}_{3}, 100 \mathrm{MHz}\right) \delta{ }^{13} \mathrm{C} \mathrm{NMR}$ $\left(\mathrm{CDCl}_{3}, 100 \mathrm{MHz}\right) \delta 115.0$ (d) (C-3), 119.6 (s), 120.6 (d), 125.7 (s), 128.5 (d), 128.8 (d), 130.0 (d), 134.3 (d), 134.5 (s), 137.8 (d), 154.6 (s), 158.0 (s) (C-2), 176.9 (s) (C-4), 187.6 (s) ( $\mathrm{Ar}{ }^{\prime} \mathrm{C}=\mathrm{O}$ ); EI-MS (70 eV) $\mathrm{m} / z$ (rel. intensity, \%) 330 ([M+2] $\left.]^{+}, 15\right), 328\left(\mathrm{M}^{+}, 16\right), 302$ (50), 301 (16), 300 (50), 221 (12), 105 (100), 77 (62); Anal. calcd for $\mathrm{C}_{16} \mathrm{H}_{9} \mathrm{BrO}_{3}$ : C, 58.38; H, 2.76. Found: C, 58.32; H, 3.05 .

2-Benzoyl-6-chloro-1-benzopyran-4-one (2d). It $(0.40 \mathrm{~g}, 70 \%)$ was obtained as colorless crystals, mp $150-152{ }^{\circ} \mathrm{C}$ (lit. ${ }^{13 \mathrm{~b}} 150{ }^{\circ} \mathrm{C}$ ); $\mathrm{R}_{\mathrm{f}} 0.45$ (ethyl acetate/ $n$-hexane=1:4); IR (neat) $v_{\max }$ 3065 (w), 2924 (w), 1657 (vs), 1598 (sh), 1465 (s), 1437 (s), 1359 (m), 1271 (s), 1236 (m), 1113 (w), $984(\mathrm{~m}), 857$ (m), $726(\mathrm{~m}), 683(\mathrm{~m}) \mathrm{cm}^{-1}$; ${ }^{1} \mathrm{H} \mathrm{NMR}\left(\mathrm{CDCl}_{3}, 400 \mathrm{MHz}\right) \delta 6.89$ (s, 1H, H-3), 
7.55 (d, J=8.8 Hz, 1H, H-8), 7.54-7.58 (m, 2H, H-3' and H-5'), 7.70 (dd, J=8.8, 2.6 Hz, 1H, H7), 7.71 (tt, $J=8.4,1.2 \mathrm{~Hz}, 1 \mathrm{H}, \mathrm{H}-4$ '), 7.95-7.97 (m, 2H, H-2' and H-6'), 8.20 (d, $J=2.6 \mathrm{~Hz}, 1 \mathrm{H}$, $\mathrm{H}-5) ;{ }^{13} \mathrm{C} \mathrm{NMR}\left(\mathrm{CDCl}_{3}, 100 \mathrm{MHz}\right) \delta{ }^{13} \mathrm{C} \mathrm{NMR}\left(\mathrm{CDCl}_{3}, 100 \mathrm{MHz}\right) \delta 114.8$ (d) (C-3), 120.4 (d), 125.2 (s), 125.3 (d), 128.8 (s), 130.0 (d), 132.1 (d), 134.3 (d), 134.5 (s), 135.0 (d), 154.1 (s), 157.9 (s) (C-2), 177.0 (s) (C-4), 187.5 (s) (Ar'C=O); EI-MS (70 eV) m/z (rel. intensity, \%) 286 $\left([\mathrm{M}+2]^{+}, 7\right), 284\left(\mathrm{M}^{+}, 22\right), 283$ (12), 258 (29), 257 (19), 256 (91), 255 (15), 221 (10), 105 (100), 77 (59); Anal. calcd for $\mathrm{C}_{16} \mathrm{H}_{9} \mathrm{ClO}_{3}$ : C, 67.50; H, 3.19. Found: C, 67.23; H, 3.38.

8-Methoxy-2-(4-methoxybenzoyl)-1-benzopyran-4-one (2e). It (0.51 g, 82\%) was obtained as colorless crystals, mp $166-167^{\circ} \mathrm{C} ; \mathrm{R}_{\mathrm{f}} 0.31$ (ethyl acetate $/ n$-hexane=1:2); IR (neat) $v_{\max } 3072(\mathrm{w})$, 2927 (w), 1646 (vs), 1599 (vs), 1575 (s), 1491 (m), 1384 (m), 1257 (s), 1178 (s), 1152 (m), 1056 (m), $1025(\mathrm{~m}), 908(\mathrm{~m}), 850(\mathrm{~m}), 744(\mathrm{~m}) \mathrm{cm}^{-1} ;{ }^{1} \mathrm{H} \mathrm{NMR}\left(\mathrm{CDCl}_{3}, 400 \mathrm{MHz}\right) \delta 3.93(\mathrm{~s}, 3 \mathrm{H}$, $\left.\mathrm{OCH}_{3}\right), 4.00$ (s, 3H, OCH 3$), 6.92$ (s, 1H, H-3), 7.02 (d, J=9.2 Hz, 2H, H-3'and H-5'), 7.24 (dd, $J=8.0,1.4 \mathrm{~Hz}, 1 \mathrm{H}, \mathrm{H}-7), 7.39$ (t, $J=8.0 \mathrm{~Hz}, 1 \mathrm{H}, \mathrm{H}-6), 7.79$ (dd, $J=8.0,1.4 \mathrm{~Hz}, 1 \mathrm{H}, \mathrm{H}-5), 8.14$ (d, $J=9.2 \mathrm{~Hz}, 2 \mathrm{H}, \mathrm{H}-2$ ' and H-6'); ${ }^{13} \mathrm{C} \mathrm{NMR}\left(\mathrm{CDCl}_{3}, 100 \mathrm{MHz}\right) \delta{ }^{13} \mathrm{C} \mathrm{NMR}\left(\mathrm{CDCl}_{3}, 100 \mathrm{MHz}\right) \delta$ 55.6 (q), 56.4 (q), 113.9 (d), 114.0 (d), 115.0 (d) (C-3), 116.4 (d), 125.5 (s), 125.6 (d), 127.4 (s), 133.0 (d), 146.3 (s), 149.3 (s), 158.6 (s) (C-2), 164.6 (s), 178.3 (s) (C-4), 185.3 (s) (ArC=O); EIMS (70 eV) m/z (rel. intensity, \%) $310\left(\mathrm{M}^{+}, 27\right), 281$ (8), 136 (9), 135 (100), 107 (9), 77(15); Anal. Calcd for $\mathrm{C}_{18} \mathrm{H}_{14} \mathrm{O}_{5}$ : C, 69.67; H, 4.55. Found: C, 69.27; H, 4.59.

7-Methoxy-2-(4-methoxybenzoyl)-1-benzopyran-4-one (2f). It (0.53 g, 85\%) was obtained as a colorless crystals, mp $178{ }^{\circ} \mathrm{C}$; $\mathrm{R}_{\mathrm{f}} 0.38$ (ethyl acetate/ $n$-hexane=1:2); IR (neat) $v_{\max } 2925(\mathrm{w})$, 1648 (s), 1600 (s), 1507 (w), 1437 (m), 1382 (w), 1257 (vs), 1169 (m), 1023 (w), 842 (w), 767 (w) $\mathrm{cm}^{-1} ;{ }^{1} \mathrm{H} \mathrm{NMR}\left(\mathrm{CDCl}_{3}, 400 \mathrm{MHz}\right) \delta 3.92\left(\mathrm{~s}, 6 \mathrm{H}, 2 \times \mathrm{OCH}_{3}\right), 6.78(\mathrm{~s}, 1 \mathrm{H}, \mathrm{H}-3), 6.96(\mathrm{~d}$, $J=2.4 \mathrm{~Hz}, 1 \mathrm{H}, \mathrm{H}-8), 7.02$ (d, $J=9.0 \mathrm{~Hz}, 2 \mathrm{H}, \mathrm{H}-3$ ' and H-5'), 7.03 (dd, J=8.8, $2.4 \mathrm{~Hz}, 1 \mathrm{H}, \mathrm{H}-6$ ), 7.99 (d, $J=9.0 \mathrm{~Hz}, 2 \mathrm{H}, \mathrm{H}-2$ ' and H-6'), 8.14 (d, $J=8.8 \mathrm{~Hz}, 1 \mathrm{H}, \mathrm{H}-5) ;{ }^{13} \mathrm{C} \mathrm{NMR}\left(\mathrm{CDCl}_{3}, 100 \mathrm{MHz}\right)$ $\delta 55.6$ (q), 55.9 (q), 100.5 (d), 114.1 (d), 114.8 (d) (C-3), 115.5 (s), 118.4 (s), 127.1 (d), 127.5 (d), 132.7 (d), 157.8 (s), 158.1 (s) (C-2), 164.6 (s), 164.8 (s), 177.4 (s) (C-4), 186.2 (s) $($ Ar'C $=\mathrm{O})$; EI-MS (70 eV) m/z (rel. intensity, \%) $310\left(\mathrm{M}^{+}, 46\right), 282$ (34), 281 (39), 135 (100), 107 (11), 77 (26); Anal. calcd for $\mathrm{C}_{18} \mathrm{H}_{14} \mathrm{O}_{5}$ : C, 69.67; H, 4.55. Found: C, 69.56; H, 4.59.

2-Benzoyl-6,8-dichloro-1-benzopyran-4-one (2g). It (0.22 g, 35\%) was obtained as a colorless crystals, mp $132-133{ }^{\circ} \mathrm{C} ; \mathrm{R}_{\mathrm{f}} 0.58$ (ethyl acetate $/ n$-hexane $\left.=1: 4\right) ; \mathrm{IR}$ (neat) $v_{\max } 3073(\mathrm{w}), 1661$ (vs), 1595 (m), 1562 (w), 1446 (s), 1355 (m), 1319 (w), 1265 (s), 1194 (w), 1131 (w), 1106 (w), 987 (m), 863 (s), 723 (s) $\mathrm{cm}^{-1} ;{ }^{1} \mathrm{H} \mathrm{NMR}\left(\mathrm{CDCl}_{3}, 400 \mathrm{MHz}\right) \delta 7.02$ (s, 1H, H-3), 7.55-7.59 (m, 2H, H-3' and H-5'), 7.71 (tt, $J=7.6,1.2 \mathrm{~Hz}, 1 \mathrm{H}, \mathrm{H}-4$ '), 7.81 (d, $J=2.6 \mathrm{~Hz}, 1 \mathrm{H}, \mathrm{H}-7$ ), 8.06-8.09 (m, 2H, H-2' and H-6'), 8.12 (d, J=2.6 Hz, 1H, H-5); $\left.{ }^{13} \mathrm{C} \mathrm{NMR} \mathrm{CDCl}_{3}, 100 \mathrm{MHz}\right) \delta 114.4$ (d) (C-3), 124.0 (d), 125.1 (s), 126.3 (d), 128.8 (s), 130.3 (s), 131.9 (s), 134.3 (d), 134.5 (d), 134.8 (d), 150.3 (s), 158.3 (s) (C-2), 176.4 (s) (C-4), 186.5 (s) (Ar'C=O); EI-MS (70 eV) m/z (rel. intensity, \%) $320\left([\mathrm{M}+2]^{+}, 20\right), 318\left(\mathrm{M}^{+}, 29\right), 292$ (46), 291 (17), 290 (71), 105 (100), 77 (78); Anal. calcd for $\mathrm{C}_{16} \mathrm{H}_{8} \mathrm{Cl}_{2} \mathrm{O}_{3}$ : C, 60.22; $\mathrm{H}, 2.53$. Found: $\mathrm{C}, 60.25 ; \mathrm{H}, 2.63$.

6-Chloro-2-(4-chlorobenzoyl)-1-benzopyran-4-one (2h). It (0.31 g, 49\%) was obtained as a colorless crystals, mp $175-176^{\circ} \mathrm{C} ; \mathrm{R}_{\mathrm{f}} 0.58$ (ethyl acetate $/ n$-hexane=1:4); IR (neat) $v_{\max } 3063(\mathrm{w})$, 
1651 (vs), 1607 (m), 1463 (m), 1437 (m), 1360 (w), 1278 (m), 1091 (m), 984 (m), 833 (m) 762 (m) $\mathrm{cm}^{-1} ;{ }^{1} \mathrm{H}$ NMR $\left(\mathrm{CDCl}_{3}, 400 \mathrm{MHz}\right) \delta 6.89(\mathrm{~s}, 1 \mathrm{H}, \mathrm{H}-3), 7.54$ (d, J=8.8 Hz, 1H, H-8), 7.54 (d, $J=8.8 \mathrm{~Hz}, 2 \mathrm{H}, \mathrm{H}-3^{\prime}$ and H-5'), 7.71 (dd, $\left.J=8.8,2.4 \mathrm{~Hz}, 1 \mathrm{H}, \mathrm{H}-7\right), 7.93$ (d, $J=8.8 \mathrm{~Hz}, 2 \mathrm{H}, \mathrm{H}-2^{\prime}$ and $\mathrm{H}-6$ ') $8.21(\mathrm{~d}, J=2.4 \mathrm{~Hz}, 1 \mathrm{H}, \mathrm{H}-5) ;{ }^{13} \mathrm{C} \mathrm{NMR}\left(\mathrm{CDCl}_{3}, 100 \mathrm{MHz}\right) \delta{ }^{13} \mathrm{C} \mathrm{NMR}\left(\mathrm{CDCl}_{3}, 100\right.$ $\mathrm{MHz}) \delta 114.7$ (d) (C-3), 120.4 (d), 125.3 (s), 129.2 (d), 129.3 (s), 131.4 (d), 132.3 (d), 132.8 (s), 135.1 (d), 141.1 (s), 154.1 (s), 157.7 (s) (C-2), 176.9 (s) (C-4), 186.3 (s) (Ar'C=O); EI-MS (70 eV) $m / z$ (rel. intensity, \%) $320\left([\mathrm{M}+2]^{+}, 10\right), 318\left(\mathrm{M}^{+}, 15\right), 292$ (17), 290 (26), 141 (32), 139 (100), 111 (30), 75 (14); Anal. calcd for $\mathrm{C}_{16} \mathrm{H}_{8} \mathrm{Cl}_{2} \mathrm{O}_{3}$ : C, 60.22; H, 2.53. Found: C, 59.94; $\mathrm{H}$, 2.69 .

6-Chloro-2-(4-methoxybenzoyl)-1-benzopyran-4-one (2i). It (0.41 g, 65\%) was obtained as a colorless crystals , mp $116-117{ }^{\circ} \mathrm{C}$ (lit. ${ }^{13 \mathrm{~b}} 114{ }^{\circ} \mathrm{C}$ ); $\mathrm{R}_{\mathrm{f}} 0.35$ (ethyl acetate/ $n$-hexane $=1: 4$ ); IR (neat) $v_{\max } 1655$ (vs), 1598 (vs), 1464 (s), 1437 (s), 1359 (m), 1262 (vs), 1174 (s), 1026 (m), 983 (m), $860(\mathrm{~m}), 772(\mathrm{~m}) \mathrm{cm}^{-1} ;{ }^{1} \mathrm{H} \mathrm{NMR}\left(\mathrm{CDCl}_{3}, 400 \mathrm{MHz}\right) \delta 3.93\left(\mathrm{~s}, 3 \mathrm{H}, \mathrm{OCH}_{3}\right), 6.84(\mathrm{~s}, 1 \mathrm{H}, \mathrm{H}-3)$, 7.02 (d, $J=8.8 \mathrm{~Hz}, 2 \mathrm{H}, \mathrm{H}-3^{\prime}$ and H-5'), 7.55 (d, $\left.J=8.8 \mathrm{~Hz}, 1 \mathrm{H}, \mathrm{H}-8\right), 7.70$ (dd, $J=8.8,2.6 \mathrm{~Hz}$, $1 \mathrm{H}, \mathrm{H}-7), 7.99$ (d, $J=8.8 \mathrm{~Hz}, 2 \mathrm{H}, \mathrm{H}-2$ ' and H-6'), 8.21 (d, $J=2.6 \mathrm{~Hz}, 1 \mathrm{H}, \mathrm{H}-5) ;{ }^{13} \mathrm{C} \mathrm{NMR}\left(\mathrm{CDCl}_{3}\right.$, $100 \mathrm{MHz}) \delta 55.7$ (q), 114.21 (d), 114.23 (d) (C-3), 120.4 (d), 125.3 (d), 125.4 (s), 127.2 (s), 132.0 (d), 132.7 (d), 134.9 (s), 154.2 (s), 158.8 (s) (C-2), 164.8 (s), 177.0 (s) (C-4), 185.7 (s) $(\mathrm{ArC}=\mathrm{O})$; EI-MS (70 eV) $\mathrm{m} / z$ (rel. intensity, \%) $316\left([\mathrm{M}+2]^{+}, 5\right), 314\left(\mathrm{M}^{+}, 15\right), 286(8), 285(8)$, 136 (9), 135 (100), 107 (8), 77 (14); Anal. calcd for $\mathrm{C}_{17} \mathrm{H}_{11} \mathrm{ClO}_{4}$ : C, 64.88; H, 3.52. Found: C, 64.86; H, 3.55.

2-(4-Methoxybenzoyl)-1-benzopyran-4-one (2j). It (0.41 g, 73\%) was obtained as a colorless crystal, mp 73-74 ${ }^{\circ} \mathrm{C}$ (lit. ${ }^{13 \mathrm{~b}} 72{ }^{\circ} \mathrm{C}$ ); $\mathrm{R}_{\mathrm{f}} 0.20$ (ethyl acetate/n-hexane $=1: 5$ ); IR (neat) $v_{\max } 2929$ (w), 1652 (vs), 1597 (vs), 1463 (s), 1379 (m), 1312 (m), 1259 (vs), 1174 (s), 1135 (w), 1024 (m), $873(\mathrm{~m}), 767(\mathrm{~m}) \mathrm{cm}^{-1}$; ${ }^{1} \mathrm{H} \mathrm{NMR}\left(\mathrm{CDCl}_{3}, 400 \mathrm{MHz}\right) \delta 3.93\left(\mathrm{~s}, 3 \mathrm{H}, \mathrm{OCH}_{3}\right), 6.85(\mathrm{~s}, 1 \mathrm{H}, \mathrm{H}-3), 7.02$ (d, $J=8.8 \mathrm{~Hz}, 2 \mathrm{H}, \mathrm{H}-3$ ' and H-5'), 7.48 (ddd, $J=8.0,7.2,0.8 \mathrm{~Hz}, 1 \mathrm{H}, \mathrm{H}-6), 7.58$ (dd, $J=8.8,0.8$ Hz, 1H, H-8), 7.76 (ddd, J=8.8, 7.2, 1.0 Hz, 1H, H-7), 8.01 (d, $J=8.8$ Hz, 2H, H-2' and H-6'), 8.25 (d, $J=8.0,1.0 \mathrm{~Hz}, 1 \mathrm{H}, \mathrm{H}-5) ;{ }^{13} \mathrm{C} \mathrm{NMR}\left(\mathrm{CDCl}_{3}, 100 \mathrm{MHz}\right) \delta 55.7$ (q), 114.1 (d), 114.3 (d) (C-3), 118.6 (d), 124.5 (s), 125.8 (d), 125.9 (d), 127.3 (d), 132.7 (d), 134.6 (s), 155.8 (s), 158.7 (s) (C-2), 164.6 (s), 178.2 (s) (C-4), 186.0 (s) (Ar'C=O); EI-MS (70 eV) m/z (rel. intensity, \%) $280\left(\mathrm{M}^{+}, 46\right), 252$ (27), 251 (23), 135 (100), 107 (10), 77 (27); Anal. calcd for $\mathrm{C}_{17} \mathrm{H}_{12} \mathrm{O}_{4}$ : C, 72.85; H, 4.32. Found: C, 72.57; H, 4.28.

\section{Acknowledgements}

The financial support from NSC (NSC 97-2113-M-037-001-MY2) Taiwan, ROC and the valuable comments from anonymous reviewers for improving the quality of this paper are grateful. 


\section{References}

1. (a) Mathew, L.; Sankararaman, S. J. Org. Chem. 1993, 58, 7576. (b) Oku, A.; Kinugasa, M.; Kamada, T. Chem. Lett. 1993, 1, 165.

2. Horita, K.; Yoshioka, T.; Tanaka, T.; Oikawa, Y.; Yonemitsu, O. Tetrahedron 1986, 42, 3021.

3. Bortolotti, B.; Leardini, R.; Nanni, D.; Zanardi, G. Tetrahedron 1993, 49, 10157.

4. (a) Kumar, V.; Sharma, A.; Sharma, M.; Sharma, U. K.; Sinha, A. K. Tetrahedron 2007, 63, 9718. (b) Lehr, R. E.; Kole, P. L.; Tschappat, K. D. Tetrahedron Lett. 1986, 27, 1649.

5. Joshi, B. P.; Sharma, A.; Sinha, A. K. Tetrahedron 2005, 61, 3075.

6. (a) Zhang, Y.; Li, C. J. Angew. Chem. Int. Ed. 2006, 45, 1949. (b) Zhang, Y.; Li, C. J. J. Am. Chem. Soc. 2006, 128, 4242.

7. Chang, J.; Zhao, K.; Pan, S. Tetrahedron Lett. 2002, 43, 951.

8. Tsang, A. S. K.; Todd, M. H. Tetrahedron Lett. 2009, 50, 1199.

9. Sinha, A. K.; Acharya, R.; Joshi, B. P. J. Nat. Prod. 2002, 65, 764.

10. Joshi, B. P.; Sharma, A.; Sinha, A. K. Tetrahedron 2005, 61, 3075.

11. (a) Haldar, P.; Guin, J.; Ray, J. K. Tetrahedron Lett. 2005, 46, 1071. (b) Pi, H. J.; Liu, H.; Du, W.; Deng, W. P. Tetrahedron Lett. 2009, 50, 4529. (c) Zhai, L.; Shukla, R.; Rathore, R. Org. Lett. 2009, 11, 3474. (d) Liu, L.; Floreancig, P. E. Org. Lett. 2009, 11, 3152. (e) Iranpoor, N.; Firouzabad, H.; Nowrouzi, N.; Khalili, D. Tetrahedron 2009, 65, 3893. (f) Silva, V. L. M.; Silva, A. M. S.; Pinto, Diana C. G. A.; Elguero, J.; Cavaleiro, J. A. S. Eur. J. Org. Chem. 2009, 26, 4468. (g) Zhai, L.; Shukla, R.; Wadumethrige, S. H.; Rathore, R. J. Org. Chem. 2010, 75, 4748. (h) Jing, Y.; Xu, C.-G.; Ding, K.; Lin, J.-R.; Jin, R.-H.; Tian, W.-S. Tetrahedron Lett. 2010, 51, 3242. (i) Chen, K.; Liu, C.; Deng, L.; Xu, G. Steroids 2010, 75, 513. (j) Huang, Q.; Zheng, B.-Z.; Long, Q. J. Chem. Sci. (Bangalore, India) 2010, $122,203$.

12. (a) Birt, D. F.; Hendrich, S.; Wang, W. Pharmacol. Therapeut. 2001, 90, 157. (b) Watanabe, S.; Uesugi, S.; Kikuchi, Y. Biomed. Pharmacother. 2002, 56, 302. (c) Xiao, Z. -P.; Shi, D. H.; Li, H.-Q.; Zhang, L. -N.; Xu, C.; Zhu, H. -L. Bioorg. Med. Chem. 2007, 15, 3703. (d) Chen, I. -L.; Chen, J. Y.; Shieh, P. C.; Chen, J. J.; Lee, C. H.; Juang, S. H.; Wang, T. C. Bioorg. Med. Chem. 2008, 16, 7639. (e) Yang, S. F.; Yang, W. E.; Kuo, W. H.; Chang, H. R.; Chu, S. C.; Hsieh, Y. S. Arch. Oral Biol. 2008, 53, 287. (f) Wiseman, H.; Casey, K.; Clarke, D. B.; Barnes, K. A. J. Agric. Food Chem. 2002, 50, 1404. (g) Brahmachari, G.; Gorai, D. Current Org. Chem. 2006, 10, 873.

13. (a) Schmultz, J.; Hirt, R.; Lauener, H. Helv. Chim. Acta 1952, 35, 1168. (b) Payard, M.; Tronche, P.; Bastide, J.; Bastide, P.; Chavernac, G. European J. Med. Chem. 1981, 16, 453. (c) Bratulescu, G. Acta. Chim. Slov. 2002, 49, 173.

14. Li, S. R.; Chen, L. Y.; Tsai, J. C.; Tzeng, J. Y.; Tsai, I. L.; Wang, E. C. Tetrahedron Lett. 2007, 48, 2139. 
15. Li, S. R.; Chen, P. Y.; Chen, L. Y.; Lo, Y. F.; Tsai, I. L.; Wang, E. C. Tetrahedron Lett. 2009, 50, 2121.

16. Li, S. R.; Shu, C. J.; Chen, L. Y.; Chen, H. M.; Chen, P. Y.; Wang, E. C. Tetrahedron 2009, 65,8702 .

17. Chen, L. Y.; Li, S. R.; Chen, P. Y.; Tsai, I. L.; Wang, T. P.; Wang, E. C. Tetrahedron Lett. 2009, 50, 5748.

18. Harvey, R. G.; Lee, H. J. Org. Chem. 1988, 53, 4557. 